\title{
Numerical study on the interaction between waves and twin pipelines in sandy seabed
}

\author{
Jisheng Zhang $\dagger$, Jinhai Zheng $\dagger$, Chi Zhang $\dagger$, Dong-Sheng Jeng $\ddagger$, Yakun Guo $\infty$
}

$\dagger$ State Key Laboratory of HydrologyWater Resources and Hydraulic Engineering, Hohai University, Nanjing, 210098, China

jszhang@hhu.edu.cn

jhzheng@hhu.edu.cn

zhangchi@hhu.edu.cn
† Division of Civil Engineering, University of Dundee, Dundee, DD1 $4 \mathrm{HN}$, UK

d.jeng@dundee.ac.uk $\infty$ School of Engineering, University of Aberdeen, Aberdeen, AB24 3UE, UK y.guo@abdn.ac.uk

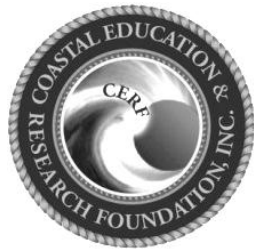

www.cerf-jcr.org

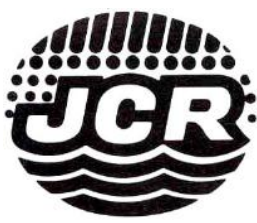

www.JCRonline.org

ABSTRACT

Zhang, J., Zheng, J., Zhang, C., Jeng, D. and Guo, Y., 2013. Numerical study on the interaction between waves and twin pipelines in sandy seabed In: Conley, D.C., Masselink, G., Russell, P.E. and O'Hare, T.J. (eds.), Proceedings $12^{\text {th }}$ International Coastal Symposium (Plymouth, England), Journal of Coastal Research, Special Issue No. 65, pp. 428433, ISSN 0749-0208.

In offshore engineering practice, the construction of an identical cylinder close to the existing pipeline may largely affect the near flow field and hydrodynamic forces on the pipelines. The interaction between incident ocean wave and twin pipelines in sandy seabed plays an important role in the design of submarine pipelines. A mathematical model based on the Volume-Averaged Reynolds-Averaged Navier-Stokes (VARANS) equations, a two-equation $k-\varepsilon$ turbulence closure and an internal source term wave maker is adopted to describe this complex phenomenon. The sandy bed where the pipelines are partly buried is treated as a rigid porous material, and its impact on wavepipeline interaction is considered in the VARANS model in terms of porosity and equivalent mean diameter of porous material. The impacts of burial depth and distance between the centers of twin pipelines on the waveseabed-twin pipelines interaction are numerically investigated. The research results from numerical simulations indicate that the burial depth and relative position of twin pipelines significantly affect the wave-averaged flow velocity and dynamic pressure distribution.

\section{ADDITIONAL INDEX WORDS: Wave model, twin pipelines, porous seabed, Navier-Stokes equations.}

\section{INTRODUCTION}

In offshore engineering, the pipeline laid on or partly buried in the porous seabed is widely used for fluid transportation, such as water intake, sewage disposal, subsea tunnel and hydrocarbons transportation. These submarine pipelines are subjected to dynamic forces due to the strong action of ocean waves. Clearly, the wave-seabed-pipeline interaction problem plays an important role in the determination of force which significantly influences the stability and performance of marine pipeline. When an identical pipeline is placed close to another (the so-called twin pipelines with tandem configuration), it may strongly disturb the flow field and wave pressure around the pipeline and consequently leads to changes in the forces on the pipelines (Cokgor and Avci, 2003).

Although the interaction between waves, seabed and a single pipeline has been extensively investigated by the coastal researchers in the recent years (Magda, 1999; Vijayakumar et al., 2003, 2005; Xu et al., 2010; Neelamani and Al-Banaa, 2012), the studies on the wave-seabed-twin pipelines are still very limited. For example, Bearman et al. (1985) investigated theoretically and experimentally the in-line forces on cylinders of general crosssection under sinusoidal flows, and evaluated corrections to the standard in-viscid inertial force at low Keulegan-Carpenter (KC) numbers. Cokgor and Avci (2003) measured the forces on a cylinder partly buried in the seabed with a parallel twin dummy cylinder nearby and without it. The forces around the cylinder in the tandem cylinder case are smaller than those in the single cylinder case, and their values largely depend on the configurations of parallel tandem cylinders and burial depth. Cokgor and Avci (2006) further performed laboratory experiments to study the hydrodynamics forces under the coexisting flow (wave and current) on the partly buried, tandem twin pipelines. The generated flow field around the pipelines (diffraction or vortex shedding regime) is dependent on the magnitude of the pipeline diameter and the incident wave characteristics, as well as on $\mathrm{KC}$ and $\mathrm{Re}$ numbers at the pipeline. All aforementioned research results indicate that the interaction between waves, porous seabed and twin pipelines is very complicated and may significantly affect the hydrodynamics forces on the pipelines.

In this study, a vertical two-dimensional mathematical model based on Volume-Averaged Reynolds-Averaged Navier-Stokes (VARANS) equations, a two-equation $k-\varepsilon$ turbulence closure and internal source term wave maker is applied to numerically study the wave propagation around and hydrodynamics force on twin pipelines in sandy seabed. In this model, the sandy seabed is treated as a rigid porous material, and it is described in terms of porosity and mean particle diameter in the governing equations. One of the advantages of this mathematical model is to describe the whole wave-seabed-twin pipelines interaction using a set of VARANS equations only. 


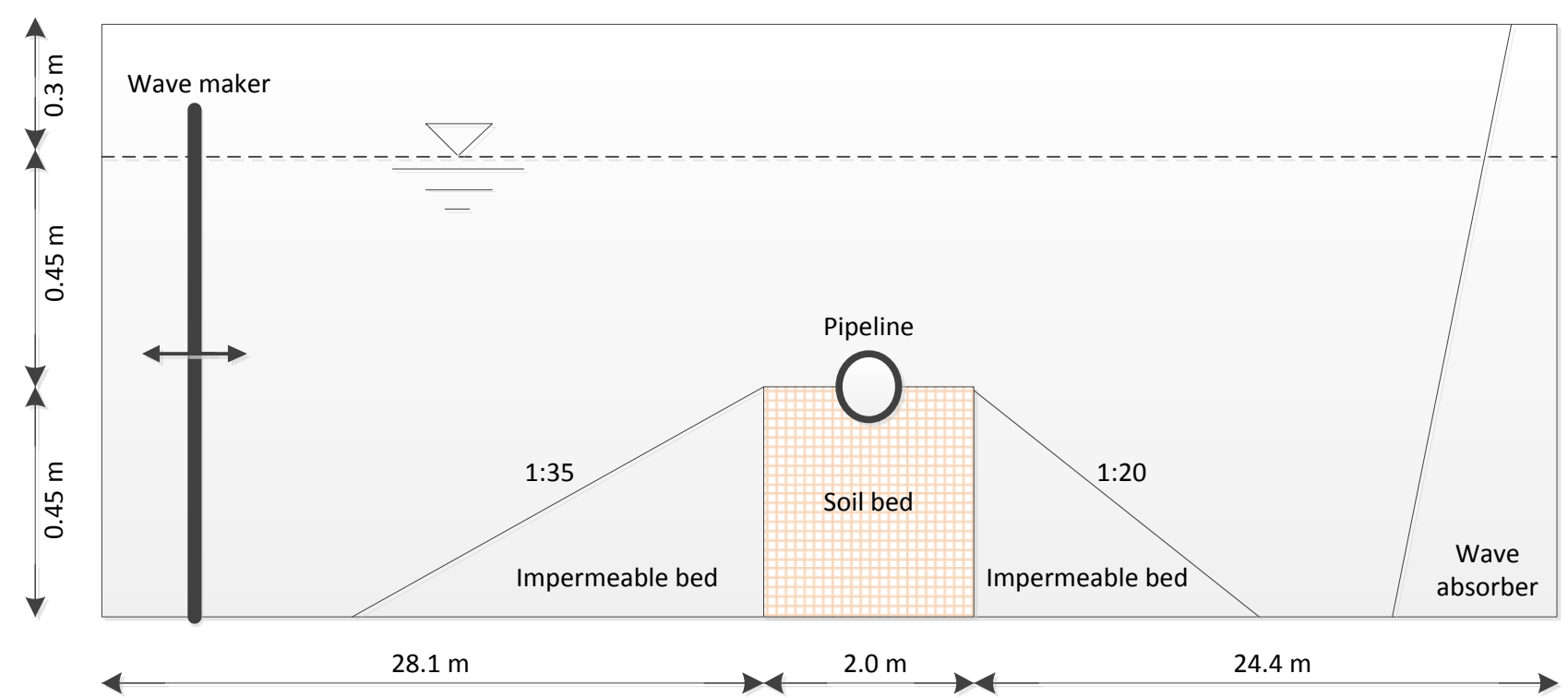

Figure 1. Sketch of laboratory experiments of Neelamani and Al-Banaa (2012)

\section{Governing Equations}

The VARANS equations for the flow motion due to waveseabed-twin pipelines can be expressed as (Hsu et al., 2002):

$$
\begin{aligned}
& \frac{\partial\left\langle\bar{u}_{i}\right\rangle}{\partial x_{i}}=0 \\
& \frac{\partial\left\langle\bar{u}_{i}\right\rangle}{\partial t}+\frac{\left\langle\bar{u}_{j}\right\rangle}{n\left(1+c_{A}\right)} \frac{\partial\left\langle\bar{u}_{i}\right\rangle}{\partial x_{j}} \\
& =\frac{1}{1+c_{A}}\left[-\frac{n}{\rho} \frac{\partial\langle\bar{P}\rangle^{f}}{\partial x_{i}}-\frac{\partial\left\langle\overline{u_{i}^{\prime} u_{j}^{\prime}}\right\rangle}{\partial x_{j}}+\frac{1}{\rho} \frac{\partial\left\langle\bar{\tau}_{i j}\right\rangle}{\partial x_{j}}+n g_{i}\right] \\
& -\frac{1}{1+c_{A}}\left[\frac{\alpha(1-n)^{2}}{n^{2} D_{50}^{2}}+\frac{\beta(1-n)}{n^{2} D_{50}} \sqrt{\left\langle\bar{u}_{1}\right\rangle^{2}+\left\langle\bar{u}_{2}\right\rangle^{2}}\right]\left\langle\bar{u}_{i}\right\rangle
\end{aligned}
$$

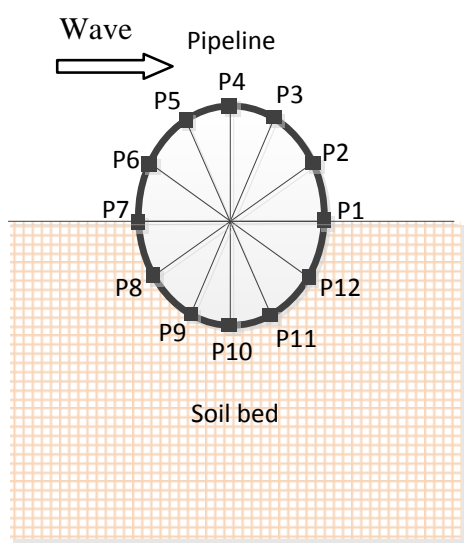

Figure 2. Distribution of pressure sensors around pipeline partly buried in soil bed. in which $x_{i}$ is Cartesian coordinate, $u_{i}$ is flow velocity, $t$ is time, $\rho$ is water density, $P$ is fluid pressure, $\tau_{i j}$ is viscous stress tensor of the mean flow, $g_{i}$ is gravitational acceleration, and $n$ and $D_{50}$ are the porosity and the equivalent mean diameter of the porous material. $c_{A}$ denotes the added mass coefficient, calculated from $c_{A}=0.34((1-n) / n) . \alpha$ and $\beta$ are empirical coefficients associated with the linear and nonlinear drag force, respectively (Lin and Liu, 1999). The influence of turbulence fluctuation on the mean flow, denoted as $\left\langle u_{i}{ }^{\prime} u_{j}{ }^{\prime}\right\rangle$, is obtained by solving the modified $k-\varepsilon$ turbulence model where $k$ is the kinetic energy and $\varepsilon$ is the dissipation rate of kinetic energy (Rodi, 1993). The over-bar represents the ensemble average and the prime denotes turbulent fluctuations with respect to the ensemble mean. The "<>", Darcy's volume averaging operator, is defined as:

$$
\langle a\rangle=\frac{1}{V} \int_{V_{f}} a d V
$$

in which $\mathrm{V}$ is the total averaging volume, and $\mathrm{V}_{\mathrm{f}}$ is the portion of $\mathrm{V}$ that is occupied by the fluid. The " $<>$ " is the intrinsic averaging operator, which is defined as:

$\langle a\rangle^{f}=\frac{1}{V_{f}} \int_{V_{f}} a d V$

The relationship between the Darcy's volume averaging operator and intrinsic volume averaging is:

$\langle a\rangle=n\langle a\rangle^{f}$

The internal source term wave maker proposed by Lin and Liu (1999) is used for the wave generation in this study.

\section{Numerical Methods}

In the model, finite difference solutions to the VARANS equations for the mean flow field and the modified $k-\varepsilon$ turbulence model for the turbulent field are obtained on a non-uniform mesh. A two-step projection method is adopted for the mean flow 
solutions, aided by the incomplete Cholesky conjugate gradient technique solving the Poisson equation for the mean pressure field. To accurately track the free water surface, the volume of fluid (VOF) method proposed by Hirt and Nichols (1981) is applied. As regards the modeling of the free surface about VOF method using a two-phase flow, this approach seems not to affect

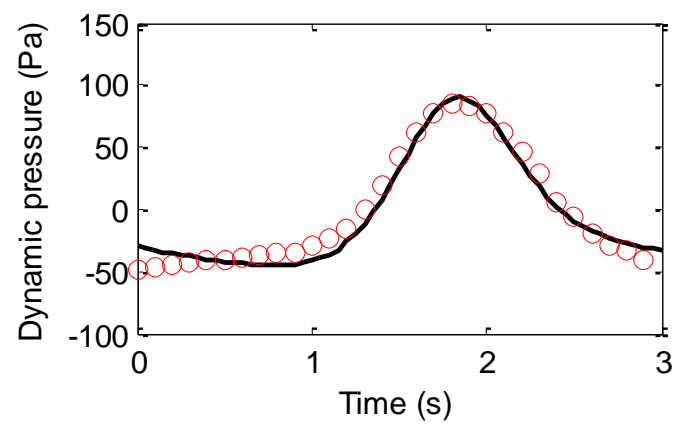

(a) P1

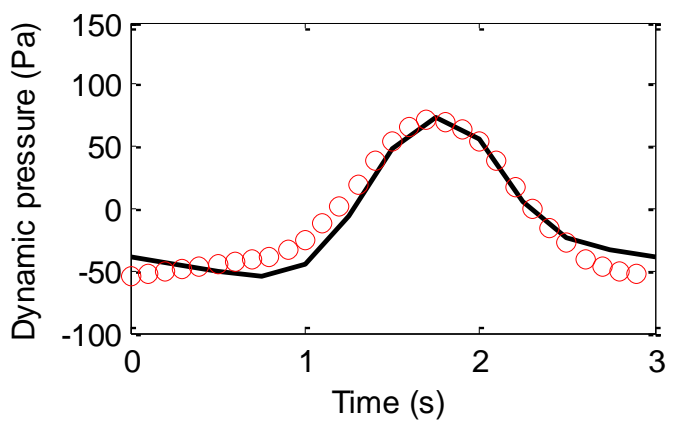

(b) $\mathrm{P} 4$

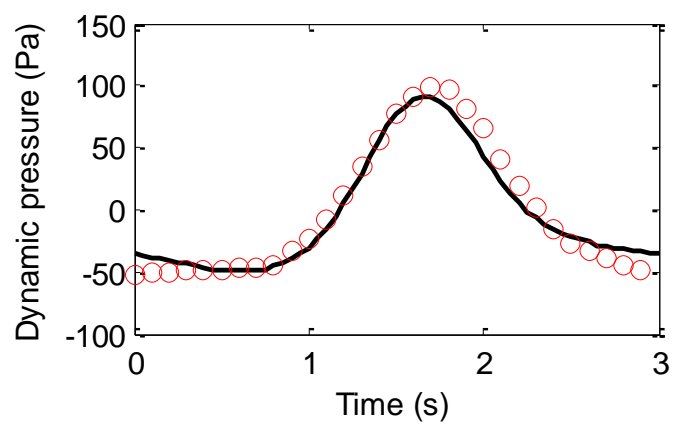

(c) P7

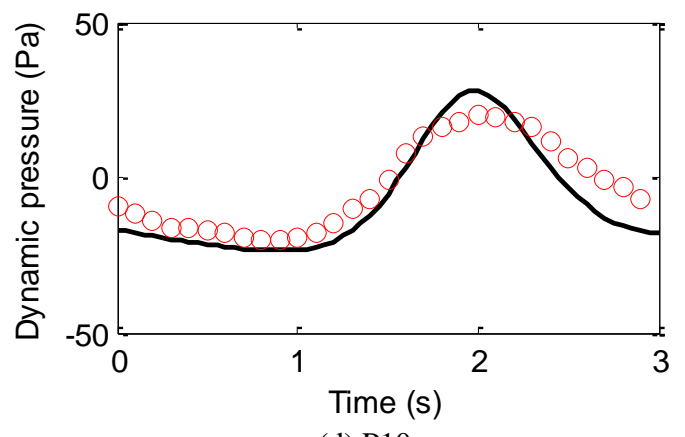

(d) P10

Figure 3. Comparisons of simulated and measured dynamic pressure at different points (a) P1, (b) P4, (c) P7 and (d) P10. o: measurement; --: simulation the flow field and hydrodynamics forces on the pipelines. The idea of VOF method is to define a function of $F$ to represent the fractional volume of water fluid. $F=1$ indicates that the cell is full of water, while $F=0$ corresponds to a cell fully occupied by air. Cells with a value of $0<F<1$ contain a water free surface, and the piecewise linear interface calculation (PLIC) method of Rider and Kothe (1998) is used to reconstruct air-water interface. The algorithm consists of two steps: a planar reconstruction of airwater interfaces within a cell; and then a geometric calculation of volume fluxes of air and water cross cell faces. In the numerical simulation, both air and water are solved.

In the simulation, appropriate boundary conditions are required. For the mean flow field, no-slip boundary condition is imposed on the sea floor surface, and the zero-stress condition is adopted on the mean free surface by neglecting the effect of air flow. For the turbulence field, the log-law distribution of mean tangential velocity in the turbulent boundary layer is applied in the grid point next to sea floor, and the zero-gradient boundary conditions are imposed for both $k$ and $\varepsilon$ on the free surface. The damping zones are located at two vertical boundaries far away from the concerned region. The detailed implementations of boundary conditions can be found in Hsu et al. (2002) and Zhang et al. (2012). To obtain the computational stability, the time interval $(\Delta t)$ during simulation is automatically adjusted at each time step to satisfy Courant-Friedrichs-Lewy condition and the diffusive limit condition (Liu et al., 1999).

\section{RESULTS AND DISCUSSION}

To validate the mathematical model, the numerical results will be compared with the experimental data of Neelamani and AlBanaa (2012). This validated model is then further used to study the effects of center distance and burial depth of twin pipelines on the distribution of dynamic pressures and flow velocity.

\section{Model Validation}

Neelamani and Al-Banaa (2012) carried out a series of physical experiments in the wave flume to investigate the wave force variation due to burial of submarine pipelines in uniformly graded and low hydraulic conductivity soil. The wave flume has a length of $54.5 \mathrm{~m}$, depth of $1.2 \mathrm{~m}$ and width of $0.6 \mathrm{~m}$. As shown in Figure 1 , the still water depth near the wave maker is $0.9 \mathrm{~m}$ and it is 0.45 $\mathrm{m}$ at the test section. The soil bed has a depth of $0.45 \mathrm{~m}$ and length of $2.0 \mathrm{~m}$. The external diameter $(D)$ of pipeline is $0.2 \mathrm{~m}$, and 12 pressure sensors are uniformly distributed around the pipeline for the measurement of wave pressure (see Figure 2 for the position of each sensor). In the experiments chosen for model validation, the main parameters are defined as: soil porosity $n=0.36$, equivalent mean diameter of soil particle $D_{50}=0.000295 \mathrm{~m}$, hydraulic conductivity of soil $k_{s}=0.000286 \mathrm{~m} / \mathrm{s}$, wave height $H_{i}=0.15 \mathrm{~m}$, wave period $T=3.0 \mathrm{sec}$, water depth $d=0.45 \mathrm{~m}$, and the ratio of pipeline burial depth to pipe diameter $e / D=0.5$.

In the numerical simulation, a computational domain with a range of $-80 \mathrm{~m} \leq x \leq 40 \mathrm{~m}$ and $0 \mathrm{~m} \leq y \leq 1.2 \mathrm{~m}$ is used. The two damping zones are located at $-80 \mathrm{~m} \leq x \leq-60 \mathrm{~m}$ and $25 \mathrm{~m} \leq x \leq 40$ $\mathrm{m}$, respectively. The original of Cartesian coordinate is located at the left sub-corner of soil bed. The cells are non-uniformly distributed in $\mathrm{x}$ direction with a minimum $\Delta x=0.005 \mathrm{~m}$ around the pipeline, while it is uniformly distributed in y direction with $\Delta y$ $=0.005 \mathrm{~m}$. The desired waves are generated at the cross section $\mathrm{x}$ $=-40 \mathrm{~m}$. In each simulation, the model was run 20 wave periods (i.e. 60 seconds), and the numerical results averaged from the last 5 wave periods are used for comparing with the laboratory experiments. 


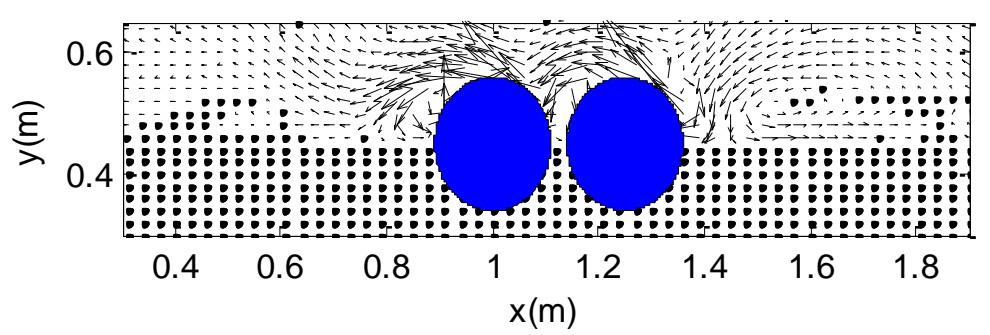

(a) Velocity field with $x / D=0.25$

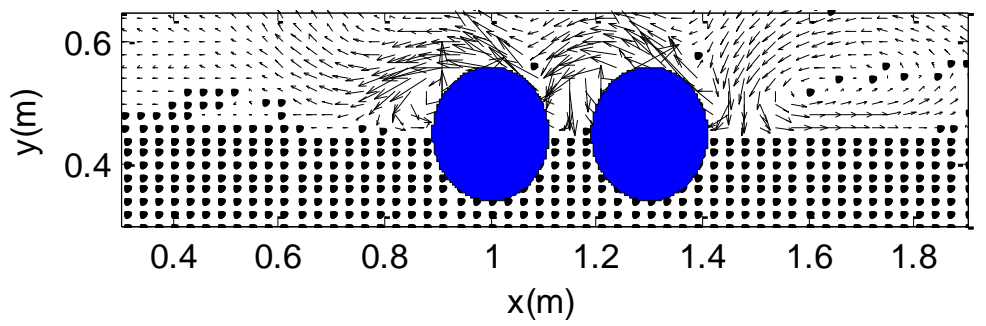

(b) Velocity field with $x / D=0.5$

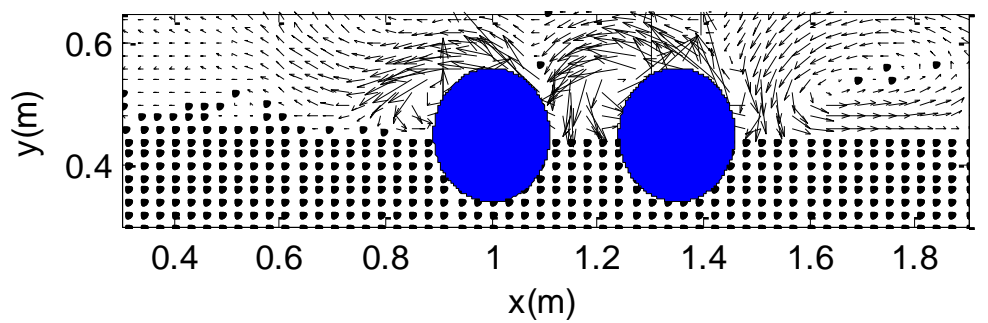

(b) Velocity field with $x / D=0.75$
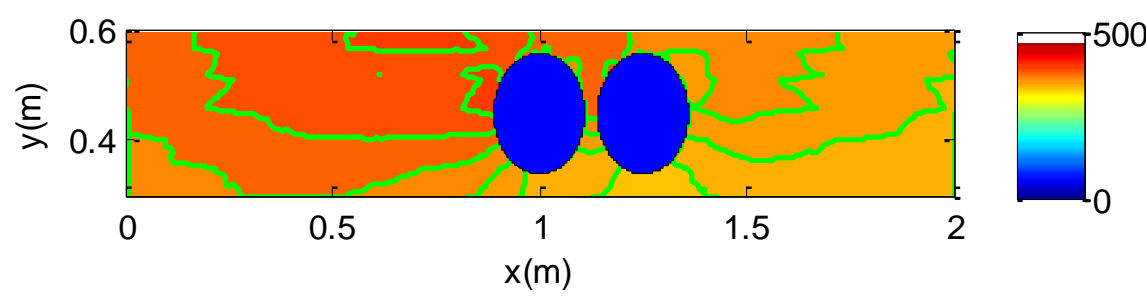

(d) Pressure field with $x / D=0.25$
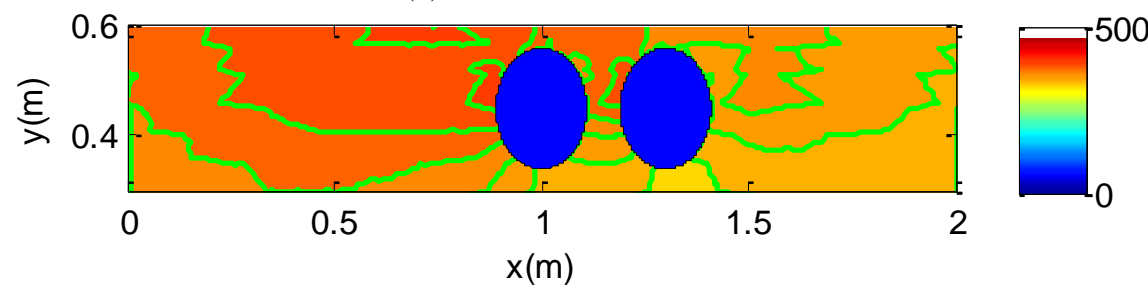

(e) Pressure field with $x / D=0.5$
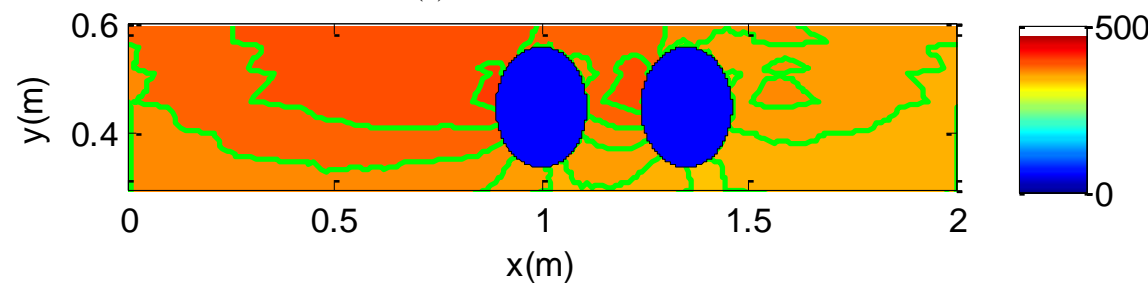

(f) Pressure field with $x / D=0.75$

Figure 4. The period-averaged velocity field and pressure field around twin pipelines with different value of $x / D$. (a-c): velocity field; (df): pressure field 


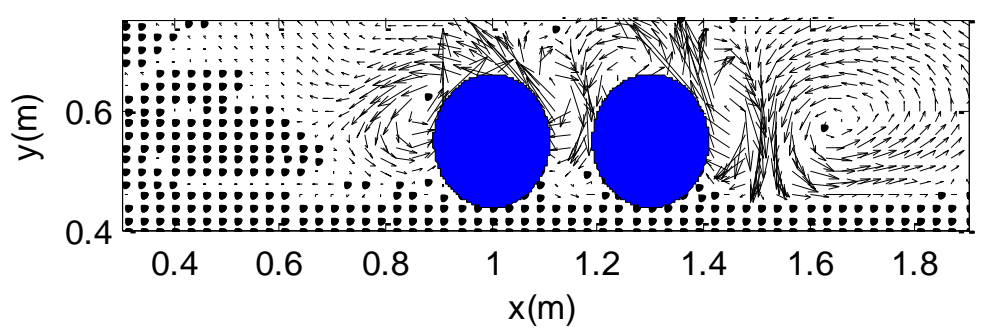

(a) Velocity field with $e / D=0$

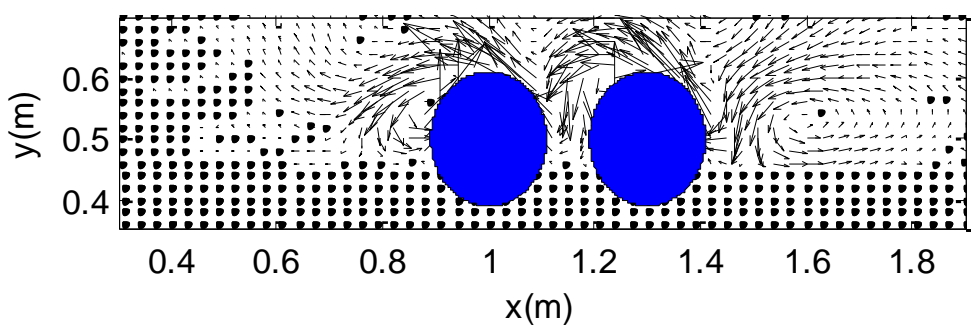

(b) Velocity field with $e / D=0.25$

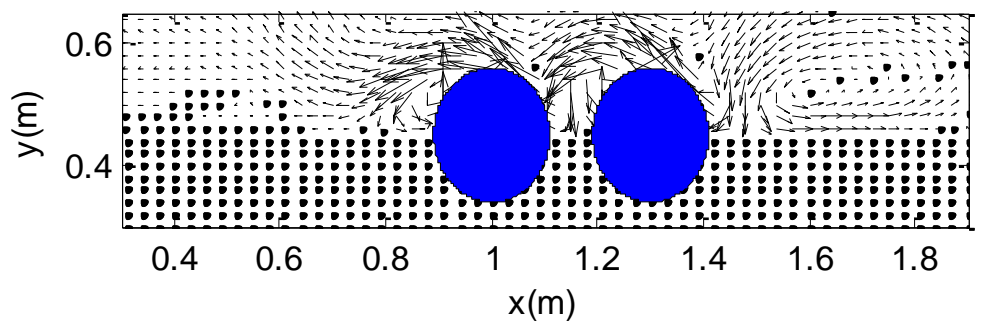

(c) Velocity field with $e / D=0.5$
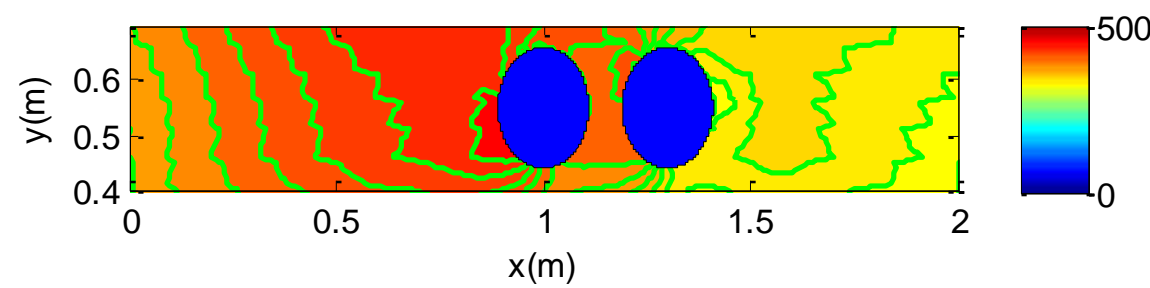

(d) Pressure field with $e / D=0$.
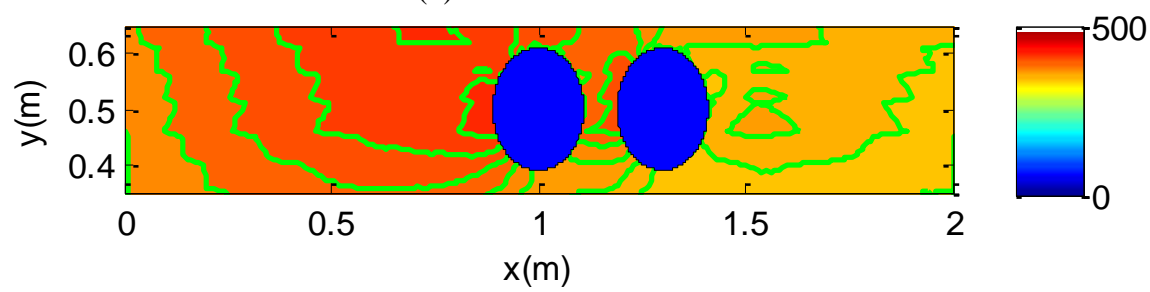

(e) Pressure field with $e / D=0.25$
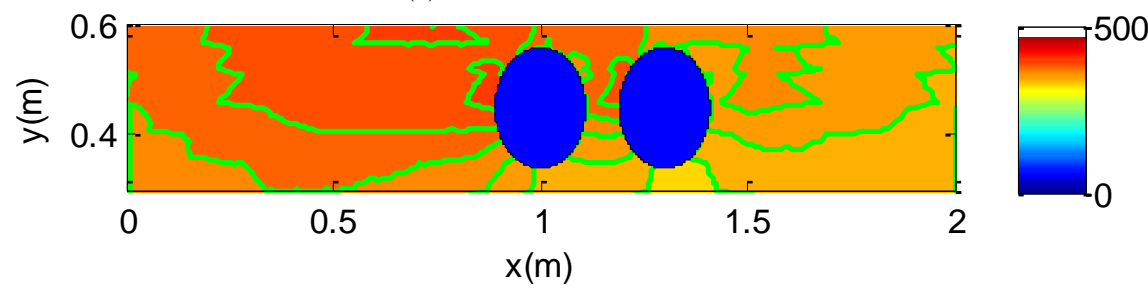

(f) Pressure field with $e / D=0.5$

Figure 5. The period-averaged velocity field and pressure field around twin pipelines with different value of $e / D$. (a-c): velocity field; (df): pressure field 
Figures 3 shows the comparisons of simulated and measured dynamic pressures at points $\mathrm{P} 1, \mathrm{P} 4, \mathrm{P} 7$ and $\mathrm{P} 10$ in the half buried case $(e / D=0.5)$, indicating a good agreement between simulation and measurement. The results indicate that the mathematical model has a great ability in predicting the complex wave-seabedpipeline interaction and the resulting dynamic pressures around the pipeline. As shown in Figure 3, the nonlinear feature of the dynamic pressures is obvious due to the interaction between waves, porous seabed and twin pipelines. As mentioned by Neelamani and Al-Banaa (2012), the difference in magnitude and phase lag between P1 and P7 is mainly responsible for the horizontal force on pipeline, while the difference in magnitude and phase lag between P4 and P10 make the main contribution to the vertical force on pipeline. It is worth noting that the horizontal and vertical forces are dependent on the contribution of several pressure sensors, as a function of the sensor arrangement sketched in Figure 2.

\section{Model Application}

In the engineering practice, the stability of marine pipeline may be enhanced by the presence of a dummy pipeline. When an identical dummy pipeline is constructed in the adjacent of the existing measurement pipeline, it may largely modify the flow field around and hydrodynamics force on the measurement pipeline. In the numerical example here, an identical dummy pipeline is replaced downstream of the existing pipeline. The distance between cylinder centers $(x / D)$ and the burial depth $(e / D)$ of these two tandem pipelines are changed to see how these configurations affect period-averaged velocity field and pressure force on the measurement pipeline.

In the example, the distance between cylinder centers $(x / D)$ are defined as $1.25,1.5$ and 1.75 , with a gap between these two pipelines are $0.25,0.5$ and 0.75 , respectively. Figure 4 shows the period-averaged velocity field and pressure field around twin pipelines. As shown in Figure 4(a-c), the velocity field in the gap between the cylinders looks complex but similar when the value of $x / D$ varies. The strong vortices can be identified both for the free side (left-hand-side) and the lee side (right-hand-side) of the dummy cylinder, as a function of $\mathrm{x} / \mathrm{D}$. The numerical results also indicate that the velocity magnitude around twin pipelines increases with an increasing $x / D$, leading to a decrease of wave pressure (see Figure 4(d-f)).

To investigate effect of the burial depth $(e / D)$ of marine pipelines on wave-seabed-twin pipelines interaction, three different value of burial depth $(e / D=0,0.25,0.5)$ are adopted in the study. The value of $e / D=0$ means that these twin pipelines are laid on the surface of seabed with zero burial depth. The periodaveraged velocity field and pressure field are shown in Figure 5. When burial depth becomes small, the space around twin pipelines for flow development is available. As shown in Figure 5(a-c), a larger vortex can be formed in the front and lee side of the tandem pipelines with a stronger velocity in the case with small burial depth. It is also noted that the wave pressure in the lee side of dummy cylinder is smaller in the case with a smaller burial depth (see Figure 5(d-f)).

It is reminded that the black points in Figures $4(\mathrm{a}-\mathrm{c})$ and 5(a-c) indicate velocity fields with a very small velocity magnitude inside porous non-movable seabed.

\section{CONCLUDING REMARKS}

In this study, the interaction between wave and twin pipelines in porous seabed has been numerically investigated by solving a set of VARANS governing equations only. This mathematical model is validated by the laboratory experiments of Neelamani and AlBanaa (2012) in term of dynamic pressures, showing a good agreement between measurement and simulation. This model is then further used to study distance between cylinder centers and burial depth of twin pipelines on the period-averaged wave velocity and pressure fields around the pipelines. Based on the numerical results, the following conclusions can be drawn.

(1) When the distance between cylinder centers varies, a complex and similar velocity field can be seen in the gap between twin pipelines. An increasing distance $\mathrm{x} / \mathrm{D}$ leads to an increasing velocity magnitude and a decreasing wave pressure around marine pipelines.

(2) The strong vortices can be identified both for the free side (left-hand-side) and the lee side (right-hand-side) of the dummy cylinder, as a function of $x / D$.

(3) When the burial depth of marine pipelines becomes smaller, a vortex with larger size and stronger velocity is formed in the front and lee side of twin pipelines, with a smaller wave pressure behind the pipelines.

\section{ACKNOWLEDGEMENT}

The authors are grateful for the support from the National Natural Science Foundation of China Grant 51209083, 51137002 and 41176073, the Natural Science Foundation of Jiangsu Province (China) Grant BK 2011026, Hohai University State Key Laboratory of Hydrology-Water Resources and Hydraulic Engineering Open Fund Scheme No 2011491311 (China).

\section{LITERATURE CITED}

Bearman, P.W., Downie, M.J., Graham, J.M.R. and Obassaju, E.D., 1985. Forces on cylinders in viscous oscillatory flow at low KeuleganCarpenter numbers. Journal of Fluid Mechanics, 154, 337-356.

Cokgor, S. and Avci, I., 2003. Forces on partly buried, tandem twin cylinders in waves at low Keulegan-Carpenter numbers. Ocean Engineering, 30(12), 1453-1466.

Cokgor, S. and Avci, I., 2006. Hydrodynamic forces on partly buried, tandem twin pipelines in coexisting flow. Journal of Coastal Research, 39, 1783-1788.

Hsu, T.J., Sakakiyama, T. and Liu, P.L.F., 2002. A numerical model for wave motions and turbulence flows in front of a composite breakwater. Coastal Engineering, 46, 25-50.

Lin, P. and Liu, P.L.F., 1999. Internal wave-maker for Navier-Stokes equations models. Journal of Waterway, Port, Coastal and Ocean Engineering, 125(4), 207-215.

Rodi, W., 1993. Turbulence models and their application in hydraulics: state-of-the-art review. IAHR, Taylor \& Francis Publisher.

Hirt, C.W. and Nichols, B.D., 1981. Volume of fluid (VOF) method for dynamics of free boundaries. Journal of Computational Physics, 39, 201-225.

Rider, W.J. and Kothe, D.B., 1998. Reconstructing volume tracking. Journal of Computational Physics, 141, 112-152.

Liu, P.L.F., Lin, P., Chang, K.A. and Sakakiyama, T., 1999. Numerical modelling of wave interaction with porous structures. Journal of Waterway, Port, Coastal and Ocean Engineering, 125(6), 322-330.

Zhang, J., Zheng, J., Jeng, D.S. and Wang, G., 2012. Numerical simulation of solitary wave induced flow motion around a permeable submerged breakwater. Journal of Applied Mathematics, 2012, Article ID 508754 , $1-14$.

Neelamani, S. and Al-Banaa, K., 2012. Wave force variation due to burial of submarine pipelines in uniformly graded and low hydraulic conductivity soil. Applied Ocean Research, 35, 47-55. 